\title{
Hemodialysis-Induced P-Wave Signal-Averaged Electrocardiogram Alterations Are Indicative of Vulnerability to Atrial Arrhythmias
}

Kentaro Shimada, MD; Takeshi Tomita, MD, PhD; Yuji Kamijo, MD, PhD; Makoto Higuchi, MD, PhD; Kenichi Ito, MD, PhD; Yoichi Koizumi, MD; Kazuya Yamamoto, MD; Kazunori Aizawa, MD, PhD; Megumi Koshikawa, MD, PhD; Hiroki Kasai, MD, PhD; Atsushi Izawa, MD, PhD; Yusuke Miyashita, MD, PhD; Setsuo Kumazaki, MD, PhD; Jun Koyama, MD, PhD; Uichi Ikeda, MD, PhD

\begin{abstract}
Background: Atrial fibrillation (AF) is the most common supraventricular arrhythmia, often occurring during hemodialysis (HD). Prolongation of the total filtered $\mathrm{P}$-wave duration (PWD) and reduction of the root mean square voltages for the last $20 \mathrm{~ms}$ of the $\mathrm{P}$ wave (RMS20) on a P-wave signal-averaged electrocardiogram (P-SAECG) are predictors of AF. We investigated whether HD induces alterations of P-SAECG, and determined the influential factors.
\end{abstract}

Methods and Results: Thirty-three end-stage kidney disease patients (66.7 \pm 12.6 years, 23 males) undergoing maintenance HD were enrolled in this study. Digital ambulatory P-SAECG monitoring and laboratory examination of serum proteins and ions were carried out before, during, and after the HD sessions. Data were analyzed by multiple regression analysis. PWD was significantly prolonged, and RMS20 significantly reduced, during HD. These values recovered after completion of HD. Multiple regression analysis showed that prolongation of PWD significantly correlated with $\mathrm{HD}$ duration and the rate of removal of body fluid. On the other hand, RMS20 significantly correlated with HD duration and blood urea nitrogen variation.

Conclusions: HD resulted in prolongation of PWD and reduction of RMS20, indicating the vulnerability of HD patients to AF. These P-SAECG changes correlated with HD duration and the rate of removal of the body fluid. These findings underline the importance of the control of dialysis variables in the prevention of atrial arrhythmias following HD. (Circ J 2012; 76: 612-617)

Key Words: Atrial fibrillation; Hemodialysis; P-wave signal-averaged electrocardiogram

A trial fibrillation (AF) is the most common supraventricular arrhythmia, often occurring in maintenance hemodialysis (HD) patients during HD sessions. ${ }^{1}$ Furthermore, AF is associated with increased incidence of cardiovascular mortality in these patients. ${ }^{2,3}$ Therefore, it is essential to understand the predisposing risk factors affecting vulnerability to AF.

The P-wave-triggered signal-averaged electrocardiogram (P-SAECG) is a noninvasive and useful technique for predicting AF. The prolongation of the total filtered P-wave duration (PWD) and the reduction of the root mean square voltages for the last $20 \mathrm{~ms}$ of the P wave (RMS20) are often detected on the
P-SAECG of paroxysmal AF patients. These ECG changes have been reported to be predictors of AF. ${ }^{4-6}$ Thus, P-SAECG is a valuable tool for assessing a patient's vulnerability to developing atrial arrhythmias. However, studies on the use of PSAECG to examine HD patients have been limited. ${ }^{7,8}$ Previous studies have compared the P-SAECG obtained before and after HD sessions, but have failed to examine a P-SAECG obtained during HD. Therefore, it is still unknown whether the HD process directly influences a patient's vulnerability to AF.

To address this question, we investigated P-SAECG alterations during the course of HD sessions. Furthermore, we analyzed the clinical parameters influencing the HD-induced P-

Received September 5, 2011; revised manuscript received November 16, 2011; accepted November 17, 2011; released online January 11, 2012 Time for primary review: 21 days

Department of Cardiovascular Medicine (K.S., T.T., K.A., M.K., H.K., A.I., Y.M., S.K., J.K., U.I.), Department of Nephrology Internal Medicine (Y. Kamijo, M.H.), Shinshu University School of Medicine, Matsumoto; Komoro Kousei Hospital, Komoro (K.I., Y. Koizumi); and Iida Municipal Hospital, Iida (K.Y.), Japan

Mailing address: Takeshi Tomita, MD, PhD, Department of Cardiovascular Medicine, Shinshu University School of Medicine, 3-1-1 Asahi, Matsumoto 390-8621, Japan. E-mail: ttomita@shinshu-u.ac.jp or Yuji Kamijo, MD, PhD, Department of Nephrology Internal Medicine, Shinshu University School of Medicine, 3-1-1 Asahi, Matsumoto 390-8621, Japan. E-mail: yujibeat@ shinshu-u.ac.jp

ISSN-1346-9843 doi:10.1253/circj.CJ-11-1000

All rights are reserved to the Japanese Circulation Society. For permissions, please e-mail: cj@j-circ.or.jp 
SAECG changes. To our knowledge, this study is the first to demonstrate increased vulnerability to $\mathrm{AF}$ development in $\mathrm{HD}$ patients, evaluated by P-SAECG alteration during HD.

\section{Methods}

\section{Patients}

From June 2008 to July 2009, end-stage kidney disease (ESKD) patients undergoing maintenance $\mathrm{HD}$ at 3 different hospitals were enrolled in this study. The protocol was in accordance with the Helsinki Declaration (2000 revision) and was approved by the institutional review board of the Shinshu University Hospital. Written informed consent was given by each patient before participation in the study.

\section{Exclusion Criteria}

The following groups of patients were excluded: not giving consent to participate in the study; ECG revealed atrial flutter, $\mathrm{AF}$, sick sinus syndrome, or atrial pacing; experienced an arrhythmogenic acute situation such as coronary syndrome, postoperative period, or infection; P-SAECG data were illegible because of severe noise level $(\geq 0.3 \mu \mathrm{V})$.

\section{HD Session Procedures}

Enrolled patients underwent HD therapy (2 or 3 times/week). High-performance hollow fiber dialyzers (composed of cellulose triacetate, polysulfone, polyacrylonitrile, and polymethyl methacrylate) were used. Anticoagulant agents used during HD sessions were heparin sodium, low-molecular-weight heparin, or nafamostat mesylate. Eight patients were administered vasopressor agents, such as amezinium metilsulfate or etilefrine hydrochloride, during the HD session.

\section{P-SAECG Procedure}

All patients underwent ambulatory ECG monitoring during the HD session by means of a Spider View Digital Holter recorder (ELA Medical, Sorin Group, Paris, France). Monitoring was started more than $30 \mathrm{~min}$ before the HD session and ended more than $30 \mathrm{~min}$ after the session. Total recording time was $1,400 \mathrm{~min}$. Data were recorded at a $1-\mathrm{kHz}$ sampling rate. We divided the recording data of the Holter ECG into 30-min periods, which were used to build the P-SAECG with the help of the Holter scanning system and SyneScope software (ELA Medical). We modulated the automatically calculated signalaveraging data to reduce the noise of the P-SAECG to a minimum. The noiseless P-SAECG data obtained at each of the 3 different phases of the HD session (before-, during-, and after) were used for analyses. Variable $\mathrm{P}$ waves were measured according to a previously described method. ${ }^{9}$ ECG data were digitized using 3 modified bipolar orthogonal leads. Signals were amplified 10,000 times, and bandpass filtering was applied to values between 1 and $300 \mathrm{~Hz}$. P waves were aligned by using the modulus difference algorithm. The highly matched $\mathrm{P}$-wave template was selected, and its $\mathrm{P}$-wave number was adjusted to an ideal state (150-200 signals) and used for signal averaging. After signal averaging, the PWD and RMS20 were automatically calculated by the Synescope software.

\section{Laboratory Data of HD Patients}

We obtained blood samples from the HD patients undergoing ambulatory ECG monitoring at each of the 3 phases of the HD session (before-, during-, and after). The relationship between P-SAECG and laboratory data, including hemoglobin $(\mathrm{Hb})$ levels, $\mathrm{pH}$, and serum concentrations of albumin (Alb), blood urea nitrogen $(\mathrm{BUN})$, creatinine $(\mathrm{Cre})$, sodium $(\mathrm{Na})$, potassium

\begin{tabular}{|c|c|c|c|}
\hline & & \multicolumn{2}{|c|}{$P$ value } \\
\hline & & $\begin{array}{l}\text { With } \\
\text { PWD }\end{array}$ & $\begin{array}{c}\text { With } \\
\text { RMS20 }\end{array}$ \\
\hline$M / F$ & $23 / 10$ & NS & NS \\
\hline Age (years) & $66.7 \pm 12.6$ & NS & NS \\
\hline \multicolumn{4}{|l|}{ Primary disease of ESRD } \\
\hline Diabetic nephropathy & 17 & NS & NS \\
\hline Hypertensive nephropathy & 3 & NS & NS \\
\hline Renal amyloidosis & 2 & NS & NS \\
\hline Chronic glomerulonephritis & 1 & NS & NS \\
\hline Post-renal acute renal failure & 1 & NS & NS \\
\hline Lupus nephritis & 1 & NS & NS \\
\hline Unknown & 8 & NS & NS \\
\hline \multicolumn{4}{|l|}{ Complications } \\
\hline Hypertension & $26(78.8 \%)$ & NS & NS \\
\hline Diabetes mellitus & $17(51.5 \%)$ & NS & NS \\
\hline Dyslipidemia & $7(21.2 \%)$ & NS & NS \\
\hline Ischemic heart disease & $9(27.3 \%)$ & NS & NS \\
\hline \multicolumn{4}{|l|}{ Drug } \\
\hline RAS inhibitor & $17(51.5 \%)$ & NS & NS \\
\hline$\beta$-blocker & $11(33.3 \%)$ & NS & NS \\
\hline Antiarrhythmic agent & $3(9.1 \%)$ & NS & NS \\
\hline Vasopressor agent & $8(24.2 \%)$ & NS & NS \\
\hline \multicolumn{4}{|l|}{ Ultrasound cardiography } \\
\hline $\operatorname{LAD}(\mathrm{cm})$ & $4.1 \pm 0.7$ & NS & NS \\
\hline LVDd (cm) & $5.0 \pm 0.7$ & NS & NS \\
\hline Ejection fraction (\%) & $64.0 \pm 12.0$ & NS & NS \\
\hline
\end{tabular}

Correlation coefficients between P-SAECG data and continuous variables were calculated using Pearson correlation analysis. PWD, P-wave duration; RMS, root mean square voltages for the last $20 \mathrm{~ms}$ of the $P$ wave; RAS inhibitor, renin-angiotensin inhibitor including angiotensin-converting enzyme II inhibitor and angiotensin receptor blocker; LAD, left atrial diameter; LVDd, left ventricular diastolic diameter; P-SAECG, P-wave-triggered signal-averaged electrocardiogram.

$(\mathrm{K})$, chloride $(\mathrm{Cl})$, calcium $(\mathrm{Ca})$, inorganic phosphorus (iP), magnesium $(\mathrm{Mg})$, and bicarbonate $\left(\mathrm{HCO}_{3}^{-}\right)$, was determined for each of the phases.

\section{Statistical Analysis}

We used the JMP software (version 8.0.1; SAS Institute, Inc, Cary, NC, USA) for statistical analysis. Data are presented as the mean \pm standard deviation (SD). Conventional statistical analysis for paired samples was performed using repeated-measures analysis of variance (ANOVA). The correlation coefficient between 2 factors was calculated by Pearson correlation analysis. Factors that might contribute to P-SAECG alterations were analyzed by stepwise multiple regression analysis. Before performing the multiple regression analysis, we checked multicollinearity. The level of statistical significance was set at $\mathrm{P}<0.05$.

\section{Results}

\section{Baseline Characteristics of HD Patients (Table 1)}

In total, 57 ESKD patients undergoing HD were initially enrolled between June 2008 and July 2009; 24 patients were excluded from the analysis based on the exclusion criteria, thus, a total of 33 adult patients (23 males, 10 females) were consecutively investigated. The mean \pm SD of age was $66.7 \pm 12.6$ years. Causative diseases of ESKD were diabetic nephropathy 


\section{PWD}

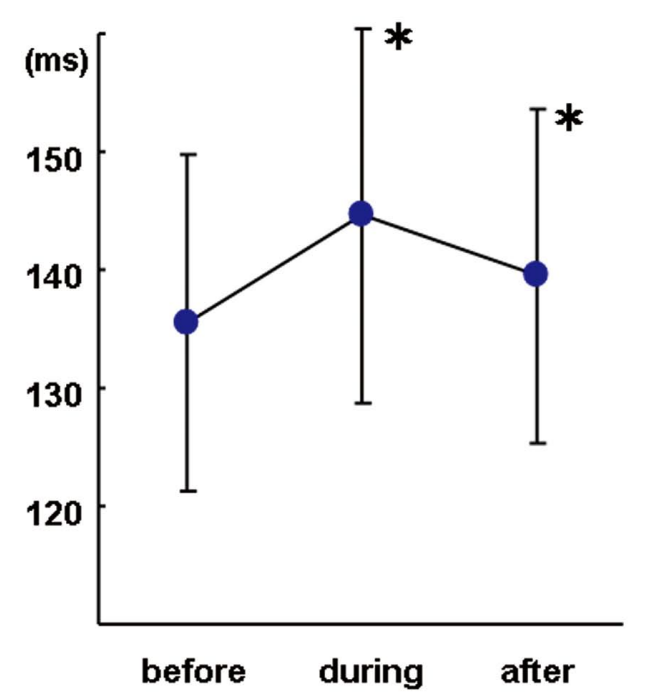

RMS20

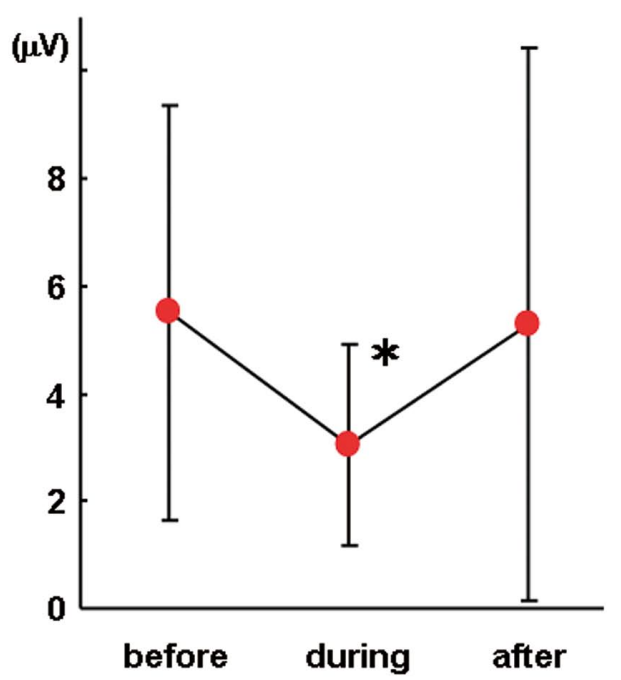

Figure. P-wave-triggered signal-averaged electrocardiogram (P-SAECG) data obtained before, during, and after hemodialysis. P-SAECG parameters, including P-wave duration (PWD) and the root mean square voltages for the last $20 \mathrm{~ms}$ of the $\mathrm{P}$ wave (RMS20), were measured before-, during-, and after the HD session $(n=33)$. PWD was markedly prolonged by the HD process, but the prolongation was attenuated after completion of the HD session. RMS20 was greatly reduced by the HD process, but fully recovered to baseline levels after the treatment. Values represent means \pm standard deviation. Significance difference of P-SAECG data from before phase is indicated with asterisks $\left({ }^{*} P<0.05\right)$.

\begin{tabular}{|lccccc|}
\hline \multicolumn{5}{|c|}{ Table 2. Alterations in Laboratory Parameters During 3 Phases of HD Session } \\
$\mathrm{Na}(\mathrm{mmol} / \mathrm{L})$ & Before & During & P value $^{\dagger}$ & \multicolumn{1}{c|}{ After } & P value $^{\ddagger}$ \\
$\mathrm{K}(\mathrm{mmol} / \mathrm{L})$ & $136.4 \pm 2.8$ & $138.0 \pm 1.8$ & $<0.0001$ & $139.0 \pm 1.5$ & $<0.0001$ \\
$\mathrm{Cl}(\mathrm{mmol} / \mathrm{L})$ & $4.3 \pm 0.8$ & $3.5 \pm 0.4$ & $<0.0001$ & $3.3 \pm 0.4$ & $<0.0001$ \\
$\mathrm{Ca}(\mathrm{mg} / \mathrm{dl})$ & $100.7 \pm 3.2$ & $102.3 \pm 2.6$ & $<0.0001$ & $102.6 \pm 2.4$ & $<0.0001$ \\
$\mathrm{iP}(\mathrm{mg} / \mathrm{dl})$ & $9.0 \pm 0.7$ & $9.3 \pm 0.6$ & $<0.0001$ & $9.5 \pm 0.6$ & $<0.0001$ \\
$\mathrm{Mg}(\mathrm{mg} / \mathrm{dl})$ & $4.2 \pm 1.2$ & $2.7 \pm 0.7$ & $<0.0001$ & $2.2 \pm 0.6$ & $<0.0001$ \\
$\mathrm{Alb}(\mathrm{g} / \mathrm{dl})$ & $2.1 \pm 0.5$ & $2.0 \pm 0.3$ & $<0.0001$ & $1.9 \pm 0.2$ & $<0.0001$ \\
$\mathrm{BUN}(\mathrm{mg} / \mathrm{dl})$ & $3.2 \pm 0.1$ & $3.1 \pm 0.1$ & $\mathrm{NS}$ & $3.1 \pm 0.1$ & $\mathrm{NS}$ \\
$\mathrm{Cre}(\mathrm{mg} / \mathrm{dl})$ & $47.1 \pm 13.2$ & $30.2 \pm 10.0$ & $<0.0001$ & $20.0 \pm 6.7$ & $<0.0001$ \\
$\mathrm{Hb}(\mathrm{g} / \mathrm{dl})$ & $8.0 \pm 2.7$ & $5.1 \pm 1.8$ & $<0.0001$ & $3.7 \pm 1.3$ & $<0.0001$ \\
$\mathrm{pH}$ & $9.5 \pm 1.8$ & $9.3 \pm 1.9$ & $\mathrm{NS}$ & $9.7 \pm 2.0$ & 0.0259 \\
$\mathrm{HCO}{ }^{-}(\mathrm{mmol} / \mathrm{L})$ & $7.41 \pm 0.04$ & $7.43 \pm 0.03$ & $<0.0001$ & $7.46 \pm 0.03$ & $<0.0001$ \\
\hline
\end{tabular}

Significant difference of each parameter from the before phase was analyzed via repeated ANOVA.

TP value between before-phase and during-phase of $H D$; $¥ P$ value between before-phase and after-phase of HD.

$\mathrm{HD}$, hemodialysis; $\mathrm{Na}$, sodium; $\mathrm{K}$, potassium; $\mathrm{Cl}$, chloride; iP, inorganic phosphorus; Mg, magnesium; Alb, albumin;

BUN, blood urea nitrogen; Cre, creatinine; $\mathrm{Hb}$, hemoglobin; $\mathrm{HCO}_{3}^{-}$, bicarbonate; ANOVA, analysis of variance.

$(\mathrm{n}=17)$, hypertensive nephropathy $(\mathrm{n}=3)$, renal amyloidosis $(n=2)$, chronic glomerulonephritis $(n=1)$, post-renal acute renal failure $(n=1)$, lupus nephritis $(n=1)$, and unknown $(n=8)$. These patients had comorbidities such as hypertension, diabetes mellitus, dyslipidemia, and ischemic heart disease. Various types of agents influencing cardiac remodeling and autonomous nerve function, including renin-angiotensin inhibitors and $\beta$ blockers, had been previously administered to these patients. Antiarrhythmic agents had been administered to 3 patients. No cases of severe atrial dilatation were detected, and cardiac systolic function was maintained within the normal range.

\section{P-SAECG Data Obtained Before, During, and After HD (Figure)}

PWD before-, during-, and after the HD session was 135.5 \pm $14.2,144.6 \pm 15.9$, and $139.5 \pm 14.1 \mathrm{~ms}$, respectively. PWD was markedly prolonged by the HD procedure, and this prolongation of PWD was attenuated after completion of the HD session. RMS20 before-, during-, and after the HD session was $5.51 \pm 3.87,3.05 \pm 1.87$, and 5.29 $\pm 5.17 \mu \mathrm{V}$, respectively. RMS20 was greatly reduced by the HD process, but it fully recovered to baseline values after the treatment. These data suggest that the HD process significantly affects P-SAECG data. On the 


\begin{tabular}{|c|c|c|c|}
\hline \multirow{3}{*}{\multicolumn{2}{|c|}{$\begin{array}{l}\text { Variation in clinical parameter } \\
\text { during HD session }\end{array}$}} & \multicolumn{2}{|c|}{ Pearson correlation } \\
\hline & & \multirow[t]{2}{*}{$\begin{array}{c}\text { With } \\
\Delta \text { PWD }\end{array}$} & \multirow[t]{2}{*}{$\begin{array}{c}\text { With } \\
\Delta \text { RMS20 }\end{array}$} \\
\hline & & & \\
\hline$\Delta \mathrm{Na}(\mathrm{mmol} / \mathrm{L})$ & $1.6 \pm 1.9$ & $0.25^{\star *}$ & $-0.23^{* *}$ \\
\hline$\Delta \mathrm{K}(\mathrm{mmol} / \mathrm{L})$ & $-0.7 \pm 0.5$ & -0.11 & $0.26^{* *}$ \\
\hline$\Delta \mathrm{Cl}(\mathrm{mmol} / \mathrm{L})$ & $1.5 \pm 1.5$ & 0.10 & $-0.27^{\star *}$ \\
\hline$\Delta \mathrm{Ca}(\mathrm{mg} / \mathrm{L})$ & $0.3 \pm 0.3$ & $0.16^{*}$ & $-0.26^{* *}$ \\
\hline$\Delta \mathrm{iP}(\mathrm{mg} / \mathrm{dl}$ & $-1.6 \pm 0.7$ & 0.02 & $0.38^{* *}$ \\
\hline$\Delta \mathrm{Mg}(\mathrm{mg} / \mathrm{dl})$ & $-0.2 \pm 0.2$ & $0.26^{\star \star}$ & -0.04 \\
\hline$\Delta \mathrm{BUN}(\mathrm{mg} / \mathrm{dl})$ & $-16.9 \pm 7.1$ & -0.12 & $0.48^{* *}$ \\
\hline$\Delta$ Cre $(\mathrm{mg} / \mathrm{dl})$ & $-2.9 \pm 1.3$ & -0.03 & $0.29^{\star *}$ \\
\hline$\Delta \mathrm{PH}$ & $0.02 \pm 0.02$ & -0.04 & -0.03 \\
\hline$\Delta \mathrm{HCO}_{3}^{-}(\mathrm{mmol} / \mathrm{L})$ & $2.7 \pm 1.4$ & 0.04 & $-0.33^{* *}$ \\
\hline \multicolumn{4}{|c|}{ Variations of P-SAECG data } \\
\hline$\Delta \mathrm{PWD}(\mathrm{ms})$ & $9.3 \pm 5.7$ & 1 & $-0.51^{* *}$ \\
\hline$\Delta \mathrm{RMS} 20(\mu \mathrm{V})$ & $-2.4 \pm 4.0$ & $-0.51^{\star *}$ & 1 \\
\hline
\end{tabular}

$\Delta$ indicates variation of each parameter between during-phase and before-phase of HD. Significance probability is indicated with asterisks $\left({ }^{*} \mathrm{P}<0.05,{ }^{* *} \mathrm{P}<0.01\right)$.

Abbreviations see in Tables1,2.

other hand, termination of the HD process had positive effects on P-SAECG data.

Serum Protein and Ion Levels Before, During, and After HD Laboratory parameters analyzed before-, during-, and after HD are shown in Table 2. Serum levels of $\mathrm{Na}, \mathrm{Cl}, \mathrm{Ca}, \mathrm{PH}$, and $\mathrm{HCO}_{3}{ }^{-}$significantly increased, whereas those of $\mathrm{K}, \mathrm{iP}, \mathrm{Mg}$, BUN, and Cre significantly decreased, in a time-dependent manner as a consequence of the HD procedure $(\mathrm{P}<0.05) . \mathrm{Hb}$ and Alb levels remained largely unchanged over the 3 collection phases.

\section{Identification of Factors Correlating With P-SAECG Alterations} We found that HD significantly affects P-SAECG and laboratory parameters. Next, we conducted Pearson correlation analysis to identify candidate factors influencing P-SAECG data (Tables 3,4). Significant candidates correlating with $\triangle P W D$ ( $\triangle \mathrm{PWD}$, where $\Delta$ indicates the variation between during- and before the HD session) were $\Delta \mathrm{Na}, \Delta \mathrm{Ca}$, and $\Delta \mathrm{Mg}$, as well as various factors inherent to the HD procedure, including the duration of HD, rate of removal of body fluid, and blood flow rate. Significant candidates correlating with $\triangle \mathrm{RMS} 20$ were $\Delta \mathrm{Na}, \Delta \mathrm{K}, \Delta \mathrm{Cl}, \Delta \mathrm{Ca}, \Delta \mathrm{PP}, \Delta \mathrm{BUN}, \Delta \mathrm{Cre}, \Delta \mathrm{HCO}_{3}^{-}$, the duration of $\mathrm{HD}$, and blood flow rate $(\mathrm{P}<0.05)$.

\begin{tabular}{|c|c|c|c|}
\hline \multirow[b]{2}{*}{ HD condition } & & \multicolumn{2}{|c|}{ Pearson correlation } \\
\hline & & $\begin{array}{c}\text { With } \\
\Delta \text { PWD }\end{array}$ & $\begin{array}{c}\text { With } \\
\Delta \text { RMS20 }\end{array}$ \\
\hline Duration of HD (months) & $29.7 \pm 43.2$ & $0.47^{\star}$ & $-0.52^{*}$ \\
\hline DW (kg) & $56.0 \pm 1.9$ & -0.11 & -0.05 \\
\hline RBF (\%DW) & $3.2 \pm 1.3$ & $0.49^{\star}$ & -0.02 \\
\hline $\mathrm{BR}(\mathrm{ml} / \mathrm{min})$ & $164.0 \pm 4.9$ & $-0.24^{*}$ & $-0.21^{*}$ \\
\hline $\operatorname{MAD}\left(\mathrm{m}^{2}\right)$ & $1.58 \pm 0.07$ & 0.00 & -0.03 \\
\hline
\end{tabular}

Significance probability is indicated with asterisks $\left({ }^{*} \mathrm{P}<0.05\right)$. DW, dry weight; RBF, rate of removal of body fluid; BR, blood flow rate; MAD, membrane area of dialyzer. Other abbreviations see in Tables1,2.

\section{Multiple Regression Analysis}

Finally, we used stepwise multiple linear regression analysis to analyze the factors that might contribute to $\triangle \mathrm{P}$-SAECG. The factors that significantly correlated with $\triangle \mathrm{PWD}$ were the rate of removal of body fluid, and duration of HD. The factors that significantly correlated with $\triangle \mathrm{RMS} 20$ were duration of HD and $\triangle \mathrm{BUN}$ (Table 5). The intensity of the correlation was indicated by the absolute values of the standardized regression coefficient and the $\mathrm{P}$ values. These findings suggest that $\Delta \mathrm{P}$ SAECG might be affected by the intensity and duration of the HD procedure.

\section{Discussion}

The present study i the first, at least to our knowledge, to examine P-SAECG data over the course of a HD session. We showed that P-SAECG data are dramatically affected as a consequence of HD. HD-induced changes, including prolongation of PWD and reduction of RMS20, were observed during (not before or after) a HD session. Remarkably, these values recovered as HD was completed, regardless of continuous variations in BUN and electrolyte values during and after HD. These findings suggested that vulnerability to atrial arrhythmias is directly and markedly exacerbated by the HD procedure. A previous study investigating another vulnerability indicator (increased P-wave dispersion) also reported a high risk of atrial arrhythmia induced by $\mathrm{HD},{ }^{10}$ thus supporting our findings. PWD significantly increased during the HD sessions compared with pre-dialysis values and then decreased at the completion of HD. The HD procedure results in drastic changes in hemodynamic conditions (decrease in atrial pressure, increase in osmotic gradient, etc). These dynamic influences result in P-SAECG changes through the alteration of the autonomic nervous system and wall stretch. These electrophysiological evidences are very consistent with the empirical

\begin{tabular}{|lcccc|}
\hline \multicolumn{2}{|l|}{ Table 5. Multiple Linear Regression Analysis } & & & \\
\\
$\quad$ PWD & B & SD & $\boldsymbol{\beta}$ & P value \\
$\quad$ Rate of removal of body fluid & 1.801073 & 0.674642 & 0.400583 & 0.0121 \\
$\quad$ Duration of HD & 0.0479365 & 0.019695 & 0.365208 & 0.0211 \\
$\quad \begin{array}{l}\text { RMS20 } \\
\text { Duration of HD }\end{array}$ & -0.038317 & 0.014227 & -0.41069 & 0.0115 \\
$\quad \Delta$ BUN & 0.193978 & 0.086332 & 0.342618 & 0.0322 \\
\hline
\end{tabular}

$\mathrm{B}$, regression coefficient; SD, standard deviation; $\beta$, standardized regression coefficient. 
clinical course of HD patients, who are reportedly more prone to atrial arrhythmias, such as AF, following HD. Atrial arrhythmias frequently induce the dysdialysis syndrome and contribute to the poor prognosis of HD patients. Therefore, understanding the causative factors influencing P-SAECG data and the development of preventive strategies based on electrophysiology are of great importance. Multiple regression analysis revealed that a longer duration of the HD treatment significantly correlated with the alteration of both PWD and RMS20. In addition to HD duration, the rate of removal of body fluid significantly correlated with the prolongation of PWD, and variation of BUN significantly correlated with the reduction of RMS20. On the other hand, the serum potassium level did not. These findings suggest that the intensity of HD affecting body fluid volume and solute removal, as well as duration of the HD procedure, might exacerbate vulnerability to atrial arrhythmias.

Several previous studies have reported that compared with non-dialysis patients, the baseline P-SAECG data of HD patients deteriorates as a function of the duration of HD. Moreover, the present study demonstrated that the P-SAECG data of patients undergoing long-term HD treatment was remarkably altered during the HD session. It is known that the clinical condition of HD patients becomes gradually aggravated following a long clinical course. ${ }^{11}$ First, the ability to regulate internal homeostasis, such as mineral and body fluid volume balance, gradually decreases in these patients because of the loss of residual renal function. Second, the incidence of ischemic heart disease of these patients is increased. Third, these patients are continuously exposed to various internal stresses, such as oxidative stress, inflammatory response, and activation of the renin-angiotensin system. ${ }^{12}$ These various situations might cause structural remodeling of the atrial muscle, promotion of fibrosis, and formation of a substrate for atrial arrhythmia, reflected in aggravation of the P-SAECG data. ${ }^{13-15}$ Additionally, it has been reported that oxidative stress induces pulmonary vein burst firing, which might be a trigger for atrial arrhythmia. ${ }^{16}$

The present study also demonstrated a significant correlation between the prolongation of PWD and the rate of removal of body fluid, as well as that between the reduction in RMS20 and the variation in BUN. High-speed removal of body fluid, which exceeds that of plasma refilling, is known to induce a decrease in intravascular volume, leading to dialysis hypotension. HD therapy generally improves unbalanced electrolytes and body fluid homeostasis. However, our results indicated that excessive, high-speed removal of body fluids might adversely affect PWD through plasma volume reduction. Several authors have pointed out that HD-induced rapid decrease in atrial pressure affected the atrial refractory phase. ${ }^{17-21} \mathrm{We}$ could not evaluate the atrial effective refractory period by electrophysiological study. Solyu et al reported that the atrial effective refractory period shortened and the atrial effective refractory period dispersion increased under high atrial pressure in patients with mitral valve stenosis. ${ }^{21} \mathrm{HD}$ patients are also exposed to high atrial pressure before completion of HD. Therefore, HD-induced atrial conduction heterogeneity (shortening of the atrial effective refractory period before completion of HD and prolongation of PWD during HD) may increase arrhythmic vulnerability and cause AF in patients with HD.

The variation in BUN is clinically utilized as a dialysis efficiency marker of solute removal. Therefore, the extent of solute removal might influence the reduction in RMS20 during the HD process. Urea nitrogen is distributed equally over the intra- cellular and extracellular fluids (interstitial fluid and plasma). On the other hand, delayed urea transfer from the intracellular to extracellular fluid appears during HD. The mechanism of delayed urea removal is related to intercompartmental clearance because of cell membrane permeability, and to regional blood flow, which differs among organs. ${ }^{22}$ Because urea nitrogen is an important osmotic substance, acute removal of urea from the circulating plasma via HD might generate a reverse osmotic gradient, which promotes the intracellular movement of water, followed by cell edema. This phenomenon is particularly obvious in the brain and is the cause of dialysis disequilibrium syndrome. ${ }^{23}$ Similarly, acute removal of urea might affect cardiomyocyte intracellular homeostasis, resulting in the reduction in RMS20. Our results indicate the importance of establishing preventive strategies against atrial arrhythmias during the HD process. In order to prevent chronic cardiac change, accompanying a HD procedure of long duration, internal homeostasis, including body fluid volume, blood pressure, electrolyte, and acid base balance, should be strictly regulated. For this purpose, fine management of diet, pharmacotherapy, and good dialysis therapy by clinical staff are needed. In order to prevent rapid internal alterations induced by the HD process, dialysis variables (ie, blood flow rate, dialyzer area, dialysate flow rate, and dialysis time) conditioning the rate of removal of body fluid and urea nitrogen clearance should be controlled. These preventive strategies have already been empirically applied in many clinical dialysis facilities. The present study brings the electrophysiological evidence that supports those empirical methods.

\section{Study Limitations}

First, the number of patients in this study was small, because the number of patients with noisy P-SAECG data was unexpectedly high. Therefore, a larger multicenter trial is needed to confirm our results. Second, we were unable to measure the actual cardiovascular data evaluated by echocardiography, together with the P-SAECG, because the echocardiography examination itself produces P-SAECG noise. Third, we could not evaluate nerve activity because HD system-related electrical noise interfered with measurement of R-R interval variability using the Holter system. Other types of cardiovascular monitoring that scarcely interfere with the P-SAECG data would be needed to determine the relationships between cardiovascular conditions and the P-SAECG data obtained during the HD process. Fourth, we excluded patients with AF and atrial flutter, so we could not address the direct relationship between HD-induced AF episodes and changes in these Pwave parameters. In fact, PAC as a trigger for AF increased during the HD session in many patients. Further studies are required to elucidate the relationship between $\mathrm{HD}$-induced $\mathrm{AF}$ episodes and changes in these P-wave parameters during HD sessions in patients with AF. We reviewed the data for the 3 patients $(9 \%)$ who received antiarrhythmic drugs in this study. During the HD session, the patients who received antiarrhythmic drugs a showed similar deterioration in P-SAECG as that observed in other patents. We conclude that administration of antiarrhythmic drugs did not affect accuracy in this study.

\section{Conclusions}

The present study is the first to demonstrate that dramatic PSAECG alterations, specifically prolongation of PWD and reduction of RMS20, occur as a consequence of HD. These alterations reveal the increased vulnerability of HD patients to atrial arrhythmias. HD-induced P-SAECG changes correlated 
with HD duration and the intensity of dialysis, including the rate of removal of body fluid and dialysis efficiency. These results suggest that tight control of dialysis variables is key to preventing atrial arrhythmias during an HD session.

\section{Acknowledgments}

We thank the co-medical staff of the HD centers, whose support and warm encouragement were inestimably valuable throughout the course of our study.

\section{References}

1. Zebe H. Atrial fibrillation in dialysis patients. Nephrol Dial Transplant 2000; 15: 765-768.

2. Genovesi S, Valsecchi MG, Rossi E, Pogliani D, Acquistapace I, De Cristofaro V, et al. Sudden death and associated factors in a historical cohort of chronic haemodialysis patients. Nephrol Dial Transplant 2009; 24: 2529-2536.

3. Ansari N, Manis T, Feinfeld DA. Symptomatic atrial arrhythmias in hemodialysis patients. Ren Fail 2001; 23: 71-76.

4. Fukunami M, Yamada T, Ohmori M, Kumagai K, Umemoto K, Sakai A, et al. Detection of patients at risk for paroxysmal atrial fibrillation during sinus rhythm by $\mathrm{P}$ wave-triggered signal-averaged electrocardiogram. Circulation 1991; 83: 162-169.

5. Guidera SA, Steinberg JS. The signal-averaged $P$ wave duration: A rapid and noninvasive marker of risk of atrial fibrillation. J Am Coll Cardiol 1993; 21: 1645-1651.

6. Ishimoto N, Ito M, Kinoshita M. Signal-averaged P-wave abnormalities and atrial size in patients with and without idiopathic paroxysmal atrial fibrillation. Am Heart J 2000; 139: 684-689.

7. Ozcan T, Horoz M, Genctoy G, Uyar B, Camsari A, Kiykim AA. The influence of hemodialysis on P-wave signal-averaged electrocardiogram findings. Artif Organs 2007; 31: 612-616.

8. Jaroszynski AJ, Glowniak A, Sodolski T, Zaluska W, WidomskaCzekajska T, Ksiazek A. Effect of haemodialysis on signal-averaged electrocardiogram P-wave parameters. Nephrol Dial Transplant 2006; 21: $425-430$

9. Stafford $\mathrm{P}$, Denbigh $\mathrm{P}$, Vincent R. Frequency analysis of the $\mathrm{P}$ wave: Comparative techniques. Pacing Clin Electrophysiol 1995; 18: 261 270.

10. Ozben B, Toprak A, Koc M, Sumerkan M, Tanrikulu AM, PapilaTopal N, et al. P wave dispersion increases during hemodialysis ses- sions. Nephron Clin Pract 2009; 112: c171-c176.

11. Chao TF, Lin YJ, Chang HL, Lo LW, Hu YF, Tuan TC, et al. Associations between renal function, atrial substrate properties and outcome of catheter ablation in patients with paroxysmal atrial fibrillation. Circ J 2011; 75: 2326-2332.

12. Nanayakkara PW, Gaillard CA. Vascular disease and chronic renal failure: New insights. Neth J Med 2010; 68: 5-14.

13. Mihm MJ, Yu F, Carnes CA, Reiser PJ, McCarthy PM, Van Wagoner $\mathrm{DR}$, et al. Impaired myofibrillar energetics and oxidative injury during human atrial fibrillation. Circulation 2001; 104: 174-180.

14. Chung MK, Martin DO, Sprecher D, Wazni O, Kanderian A, Carner CA, et al. C-reactive protein elevation in patients with atrial arrhythmias: Inflammatory mechanisms and persistence of atrial fibrillation. Circulation 2001; 104: 2886-2891.

15. Burstein B, Nattel S. Atrial fibrosis: Mechanisms and clinical relevance in atrial fibrillation. J Am Coll Cardiol 2008; 51: 802-809.

16. Lin YK, Lin FZ, Chen YC, Cheng CC, Lin CI, Chen YJ, et al. Oxidative stress on pulmonary vein and left atrium arrhythmogenesis. Circ J 2010; 74: $1547-1556$.

17. Braunschweig F, Kjellstrom B, Soderhall M, Clyne N, Linde C. Dynamic changes in right ventricular pressures during haemodialysis recorded with an implantable haemodynamic monitor. Nephrol Dial Transplant 2006; 21: 176-183.

18. Kjellstrom B, Braunschweig F, Lofberg E, Fux T, Grandjean PA, Linde C. Changes in right ventricular pressures between hemodialysis sessions recorded by an implantable hemodynamic monitor. Am J Cardiol 2009; 103: 119-123.

19. Ravelli F, Allessie M. Effects of atrial dilatation on refractory period and vulnerability to atrial fibrillation in the isolated Langendorffperfused rabbit heart. Circulation 1997; 96: 1686-1695.

20. Tse HF, Pelosi F, Oral H, Knight BP, Strickberger SA, Morady F. Effects of simultaneous atrioventricular pacing on atrial refractoriness and atrial fibrillation inducibility: Role of atrial mechanoelectrical feedback. J Cardiovasc Electrophysiol 2001; 12: 43-50.

21. Soylu M, Demir AD, Ozdemir O, Topaloglu S, Aras D, Duru E, et al. Evaluation of atrial refractoriness immediately after percutaneous mitral balloon commissurotomy in patients with mitral stenosis and sinus rhythm. Am Heart J 2004; 147: 741 - 745 .

22. Schneditz D, Fariyike B, Osheroff R, Levin NW. Is intercompartmental urea clearance during hemodialysis a perfusion term? A comparison of two pool urea kinetic models. J Am Soc Nephrol 1995; 6: $1360-1370$.

23. Patel N, Dalal P, Panesar M. Dialysis disequilibrium syndrome: A narrative review. Semin Dial 2008; 21: 493-498. 